\title{
Kikuchi-Fujimoto Disease and Prognostic Implications
}

\author{
Sebastian Salamat ${ }^{1} \cdot$ Jacquline Chan $^{1}$ (1) $\cdot$ Karan Jolly $^{1} \cdot$ George Powell $^{2} \cdot$ Katherine Harrison $^{2} \cdot$ Sajad Ahanger $^{1}$. \\ Churunal Hari ${ }^{1}$
}

Received: 17 January 2019 / Accepted: 2 March 2019 / Published online: 19 March 2019

(c) Springer Science+Business Media, LLC, part of Springer Nature 2019

\begin{abstract}
Kikuchi-Fujimoto disease (KFD) is a rare cause of lymphadenitis seen mostly in Asian populations (Kikuchi in Nippon Ketsueki Gakkai Zasshi 35:379-80, 1972). First described in 1972, KFD is a benign and self-limiting disease characterised by lymphadenopathy, mild fever, fatigue, and leukopenia (Fujimoto in Naika 30:920-7, 1972; Lin et al. in Otolaryngol Head Neck Surg 128(5): 650-3, 2003). We present a case of a 38-year-old woman with a 6-week history of cervical lymphadenopathy. Her ultrasound scan and fine needle aspiration cytology results were inconclusive. Excisional biopsy of the lymph node confirmed presence of KFD. The aetiology of KFD is unknown; however, there is strong association with systemic lupus erythematosus (SLE). Kucukardali reported 9\% of European KFD patients and 28\% of East Asian patients had concomitant SLE (Kucukardali et al. in Clin Rheumatol 26(1):50-4, 2007). We describe a follow-up algorithm for newly diagnosed KFD cases, based on the current literature. KFD is a rare cause of cervical lymphadenopathy. It is associated with increased risk of developing SLE, therefore early diagnosis and long-term follow-up are recommended.
\end{abstract}

Keywords Kikuchi-Fujimoto disease $\cdot$ Histiocytic necrotising lymphadenitis $\cdot$ Lymph node $\cdot$ Lymphadenopathy

\section{Introduction}

Kikuchi-Fujimoto disease (KFD) is a histiocytic necrotising lymphadenitis that was first identified by two Japanese pathologists, Kikuchi and Fujimoto, in 1972 [1, 2]. KFD is a benign and self-limiting disease, characterised by lymphadenopathy, fever, fatigue, and leukopenia. Initially, a large preponderance of KFD in females as compared to males was observed; however, more recent studies found the ratio to be closer, at approximately 1.25-2:1 [3, 4]. KFD typically affects young adults, with a mean age of 20-30 years old [3].

Due to the self-limiting nature of the disease, KFD is often misdiagnosed as a viral infection, resulting in frequent under-diagnosis. The symptoms of KFD can also mimic those of more sinister conditions such as tuberculous lymphadenitis and malignant lymphoma [4]. The pathogenesis of KFD is unknown, but viral or autoimmune aetiologies,

Jacquline Chan

jacquline.chan@nhs.net

1 Department of Otolaryngology, Princess Royal Hospital, Telford, Shropshire, UK

2 Department of Pathology, Shrewsbury and Telford NHS Trust, Telford, Shropshire, UK most notably systemic lupus erythematosus (SLE), are theorized.

\section{Case Report}

A 38-year-old Afro-Caribbean female was referred to our local ear, nose and throat (ENT) neck lump clinic via a general practitioner. She presented with a 3-week history of painful swellings on the left side of her neck. She had no other associated symptoms and was a non-smoker. Drug history included levothyroxine following a thyroidectomy in 2008. She was previously diagnosed with Grave's disease in 2006. Her general health was good and she regularly participated in strenuous sports.

On examination, three mobile lymph nodes were present in the posterior triangle of the neck. The erythrocyte sedimentation rate (ESR) was raised at $49 \mathrm{~mm} / \mathrm{h}$ while other serologic tests were unremarkable. White cell count was 3.8 (normal range $3.8-11.0 \times 10^{12} / 1$ ). A full ENT examination showed no other significant clinical findings. An ultrasound scan (USS) was subsequently requested.

The USS of the neck showed multiple left posterior triangle (level V) nodes, measuring up to $9 \mathrm{~mm}$ in short diameter. 
The nodes had an echogenic centre and colour flow centrally, suggesting reactive lymph nodes. Fine needle aspiration at this time returned inconclusive results. Due to the persistent swelling for 9 weeks duration, a decision was made to biopsy a lymph node and culture for tuberculosis (TB). The culture was negative for significant microorganisms, including TB.

The biopsy was interpreted as KFD by a local pathologist. It was referred for a second opinion (Heartland's Hospital in Birmingham, United Kingdom) which confirmed the diagnosis. The biopsied lymph node contained a large area of necrosis, characterised by a conspicuous absence of granulocytes. The necrotic area was rimmed by loosely arranged histiocytoid cells with pale, often twisted/crescentic nuclei (Fig. 1). The cells demonstrated low Ki-67 proliferative activity and many co-expressed myeloperoxidase. Lymphocytes associated with the necrosis, including some still recognisable cell shadows within the necrotic zones, were mostly of T-cell type (CD3+). A fairly comparable representation of CD4+ and CD8+ cells were present. Elsewhere, away from the necrotic areas, $\mathrm{CD} 20+\mathrm{B}$-cells predominated. No microorganisms were seen on periodic acid-Schiff (PAS) or Ziehl-Neelsen (ZN) stains. The patient was referred to the respiratory team and had blood tests for lupus screening. Chest X-ray revealed no suggestion of infection, sarcoidosis, TB, or malignancy. Following the lymph node biopsy, the patient's symptoms mostly resolved. No further complications or recurrences occurred to date (6 months following diagnosis)

\section{Discussion}

There are many aetiologies of lymphadenopathy including infectious and non-infectious diseases. Infectious causes include TB, human immunodeficiency virus (HIV), Castleman's disease, brucellosis, and syphilis. Non-infectious aetiologies include autoimmune diseases [primarily systemic lupus erythematosus (SLE)], sarcoidosis, lymphoma, and KFD.

The most common examination findings associated with KFD include lymphadenopathy, fever, erythematous rashes, fatigue, and arthralgia. Patients may complain of non-specific symptoms including arthritis, weight loss, loss of appetite, hepatosplenomegaly, and sweating [4]. Laboratory investigations are frequently unremarkable and therefore carry less diagnostic value in establishing a diagnosis of KFD [5]. They may be valuable, however, in excluding entities in the differential diagnosis. Careful history taking and examination is paramount in streamlining the differential diagnosis, with excisional biopsy being the gold standard of diagnosis.

The diagnosis of KFD is made on histopathologic examination of the affected lymph node. Three stages of KFD histology have been proposed: proliferative, necrotising, and xanthomatous [6]. The defining histologic features of KFD include the presence of areas of necrosis, with concentration of apoptotic cells within the central core; neutrophils and eosinophils are not found. In the surrounding periphery, a number of cells may be identified including histiocytes, immunoblasts, and a characteristic cell type known as plasmacytoid dendritic cells [7].

KFD and its association with autoimmune diseases, including SLE, granulomatosis polyangiitis, and Grave's disease, has previously been described [8]. Its association with SLE is reported by Kucukardali et al., where the authors found SLE and KFD occurred concomitantly in $13 \%$ of cases [4]. These figures are similar to a rate of $12 \%$ as reported by Dumas et al. in 2014 [9]. KFD can occur in patients with pre-existing SLE, coexist with SLE, or evolve into SLE. SLE closely simulates the clinical and histopathologic features of KFD. Fever and lymphadenopathy are common clinical presentations in both conditions. Histologic and immunohistochemical features of SLE lymphadenopathy are, in some cases, indistinguishable from KFD. These features include
Fig. 1 a Low magnification histologic image illustrates reactive lymphoid follicles and an expanded paracortex. The necrotic areas are characterised by the absence of granulocytes. b Paracortical expansion with a proliferation of lymphocytes and a "starry-sky" pattern. Crescentic nuclei are noted
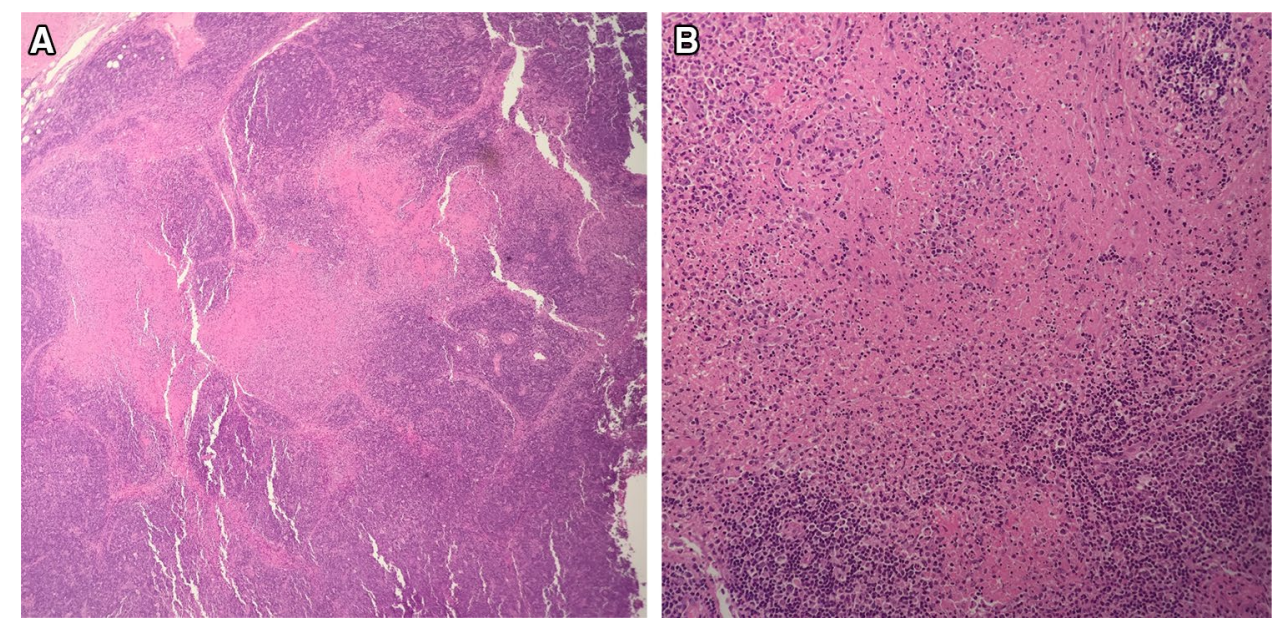
the presence of focal necrosis, absence of neutrophils and eosinophils, and co-expression of CD68 and myeloperoxidase by histiocytes [10]. Yilmaz et al. reviewed two cases of KFD that mimicked SLE and speculated that both diseases share a common hyper-immune reaction to antigenic stimuli; however, they then diverge into two distinct manifestations [11]. It appears that KFD predisposes the patient to SLE at any time, although the mean time to presentation of SLE following KFD diagnosis is difficult to pinpoint. Given the common histologic features shared by both entities, it is important to always consider SLE in the differential diagnosis.

KFD can also be easily mistaken for T-cell lymphoma. The symptoms of weight loss, fever, and sweating may be associated with both disease processes. The histologic features of lymphoma can also mimic those of KFD including the presence of immunoblasts and plasmacytoid dendritic cells [12]. Myeloperoxidase expression can help differentiate between the conditions [10].

KFD is a self-limiting condition that does not require specific management, and, consequently, there is limited literature on assessing prognostic factors for KFD. Marunaka et al. retrospectively studied $43 \mathrm{KFD}$ patients undergoing lymph node biopsy between 2001 and 2013 [13]. They found patients with low ratios of blastic cell proliferation areas $(<70 \%)$ and absence of atypical lymphocytes in peripheral blood were associated with a relatively long duration of KFD [13]. This is the only study of its kind thus far.

There is no current literature illustrating how best to follow KFD patients in regard to long-term complications and disease associations such as SLE. It appears it would be appropriate to perform an SLE screen with the initial diagnosis of KFD, followed by annual SLE screens for several years [14]. Likewise, ESR monitoring in primary care can be used to assess for any autoimmune, infectious, or malignant developments. Regardless, KFD has an excellent prognosis with most cases resolving within 6 months [13].

Three to four percent of KFD cases recur. The optimum management of patients with recurrent KFD has yet to be reported; however, case studies have suggested the concomitant use of prednisolone and hydroxychloroquine to induce remission [15-17].

\section{Conclusion}

Chronic lymph node enlargement and fever should be assessed carefully, with KFD considered as a viable diagnosis. The gold standard is histologic examination of an involved lymph node. KFD is a benign, self-limiting disease, requiring symptomatic treatment with no ongoing therapy necessary in most cases. Relapses have been reported in literature, up to many years following the first episode. Although a self-limiting condition, KFD is associated with increased risk of SLE, malignancy, and other autoimmune diseases. Therefore, early diagnosis and longterm follow-up are recommended. Following initial investigations to rule out infectious and non-infectious aetiologies, we recommend long-term follow-up in primary care, with yearly SLE screenings and ESR blood test.

\section{References}

1. Kikuchi M. Lymphadenitis showing focal reticulum cell hyperplasia with nuclear debris and phagocytes: a clinicopathological study. Nippon Ketsueki Gakkai Zasshi. 1972;35:379-80.

2. Fujimoto Y. Cervical subacute necrotizing lymphadenitis. Naika. 1972;30:920-7.

3. Lin HC, Su CY, Huang CC, Hwang CF, Chien CY. Kikuchi's disease: a review and analysis of 61 cases. OtolaryngologyHead and Neck Surg. 2003;128(5):650-3.

4. Kucukardali Y, Solmazgul E, Kunter E, Oncul O, Yildirim S, Kaplan M. Kikuchi-Fujimoto disease: analysis of 244 cases. Clin Rheumatol. 2007;26(1):50-4.

5. Ruaro B, Sulli A, Alessandri E, Fraternali-Orcioni G, Cutolo M. Kikuchi-Fujimoto's disease associated with systemic lupus erythematous: difficult case report and literature review. Lupus. 2014;23(9):939-44.

6. Amin MR. Kikuchi-Fujimoto disease-a comprehensive review. Bangladesh J Med. 2014;24(2):70-7.

7. O'Malley DP, Grimm KE. Reactive lymphadenopathies that mimic lymphoma: entities of unknown aetiology. Semin Diagn Pathol. 2013;30(2):137-45.

8. Sopena B, Rivera A, Chamorro A, Freire M, Alende V, Seco E, Gonzalez-Quintela A. Clinical association between Kikuchi's disease and systemic lupus erythematosus: a systematic literature review. Semin Arthritis Rheum. 2017;47(1):46-52.

9. Dumas G, Prendki V, Haroche J, et al. Kikuchi-Fujimoto disease: retrospective study of 91 cases and review of the literature. Medicine (Baltimore). 2014;93(24):372-82.

10. Perry AM, Choi SM. Kikuchi-Fujimoto disease. Rev Arch Pathol Lab Med. 2018;142(11):1341-6.

11. Yilmaz M, Camci C, Sari I, Okan V, Sevinc A, Onat AM, Buyukhatipoglu H. Histiocytic necrotizing lymphadenitis (KikuchiFujimoto's disease) mimicking systemic lupus erythematosus: a review of two cases. Lupus. 2006;15(6):384-7.

12. Menasce LP, Banerjee SS, Edmondson D, Harris M. Histiocytic necrotizing lymphadenitis (Kikuchi-Fujimoto disease): continuing diagnostic difficulties. Histopathology. 1998;33(3):248-54.

13. Marunaka H, Orita Y, Tachibana T, Miki K, Makino T, Gion Y, Nishizaki K, Yoshino T, Sato Y. Kikuchi-Fujimoto disease: evaluation of prognostic factors and analysis of pathologic findings. Acta Oto-Laryngol. 2016;136(9):944-7.

14. Bezek S, Tucci V, Kalra S, Fisher A. State of the globe: time to revisit Kikuchi Fujimoto disease. J Glob Infect Dis. 2014;6(4):139.

15. Honda F, Tsuboi H, Toko H, Ohyama A, Takahashi H, Abe S, Yokosawa M, Asashima H, Hagiwara S, Hirota T, Kondo Y. Recurrent Kikuchi-Fujimoto disease successfully treated by the concomitant use of hydroxychloroquine and corticosteroids. Intern Med. 2017;56(24):3373-7.

16. Castro DC, Bellido DP. Kikuchi disease: evolution during outbreaks and its response to antimalarial treatment. Revista espanola de patologia: publicacion oficial de la Sociedad 
Espanola de Anatomia Patologica y de la Sociedad. Espanola de Citologia. 2018;51(1):34-6.

17. Rezai K, Kuchipudi S, Chundi V, Ariga R, Loew J, Sha BE. Kikuchi-Fujimoto disease: hydroxychloroquine as a treatment. Clin Infect Dis. 2004;15(12):e124-6.
Publisher's Note Springer Nature remains neutral with regard to jurisdictional claims in published maps and institutional affiliations. 